\title{
Triple redundant signals effect in the visual modality*
}

\section{Efecto de señales triple redundantes en la modalidad visual}

Recibido: junio 1 de 2012 | Revisado: agosto 1 de 2012 | Aceptado: agosto 20 de 2012

\author{
SONJA ENGMANN ** \\ Université de Montréal, Canada \\ DENIS COUSINEAU *** \\ Université d'Ottawa, Canada
}

Doi:10.11144/Javeriana.UPSY12-5.trse

Para citar este artículo: Engmann, S., \& Cousineau, D. (2013). Triple redundant signals effect in the visual modality. Universitas Psychologica, 12(5), 1473. 1488. Doi:10.11144/Javeriana.UPSY12-5.trse

This research was supported by the Conseil pour la recherche en sciences naturelles et en génie du Canada, the German Academic Exchange Service (DAAD) and the Friedrich-Ebert-Stiftung.

* Université de Montréal

*** Université d'Ottawa. For correspondence: Denis Cousineau. École de Psychologie, Université d’Ottawa.E-mail: denis.cousineau@uottawa.ca

\begin{abstract}
A B S T R A C T
Response times in a visual object recognition task decrease significantly if targets can be distinguished by two redundant attributes. Redundancy gain for two attributes has been commonly found, but redundancy gain from three attributes has been found only for stimuli from three different modalities (tactile, auditory, and visual). This study extends those results by showing that redundancy gain from three attributes within the same visual modality (color, form and direction of movement) is possible. It also presents evidence that the separate activation model cannot account for such a gain. Keywords authors

Target detection; redundancy effect; triple redundancy; race model; coactivation model.

Keywords plus

Response Times, Object Recognition, Cognitive Science.

\section{RES UMEN}

Los tiempos de respuesta en las tareas de reconocimiento visual de objetos disminuye significativamente si los objetivos pueden ser distinguidos por dos atributos redundantes. La ganancia de redundancia para dos atributos se ha encontrado comúnmente, pero la ganancia de redundancia de tres atributos ha sido encontrada solo para estímulos desde tres modalidades diferentes (táctil, auditivo y visual). Este estudio se extiende a aquellos resultados mostrando que el aumento de la redundancia es posible en tres atributos dentro de la misma modalidad visual (color, forma y dirección del movimiento). También se presenta evidencia de que el modelo de activación separada no puede dar cuenta de una ganancia como tal.

Palabras clave autores

Detección de objetivos, efectos de redundancia; redundancia triple, modelo carrera, modelo coactivación.

Palabras clave descriptores

Tiempos de respuesta, reconocimiento de objetos, ciencia cognitiva.
\end{abstract}




\section{Introduction}

The environment surrounding us can be subdivided into distinct sources of information that are used to make a decision about the identity of objects. Sources of information are the different sensory modalities (e.g. auditory, visual) with different types of information within each modality (e.g. color, form, direction of motion within the visual modality). These features are processed and identified separately (although not necessarily independently) by the visual system through specialized processing channels before the object is perceived as a whole (Treisman \& Souther, 1985; Kandel, Schwartz \& Jessel, 2000; Ungerleider \& Mishkin, 1982; Milner \& Goodale, 1993).

In some cases, a single feature (e.g., the color) is enough to recognize an object. Treisman and Souther (1985) have shown that if a target object differs from several distracters by one distinct feature alone (e.g., a red square among green squares) it can be detected rapidly, accurately and without conscious effort. The detection is also independent of the number of surrounding distracters. This is known as the pop-out effect (Treisman \& Souther, 1985).

In other cases, a combination of several features is needed for an unambiguous identification. If the joint identification of two or more features is necessary to distinguish a target object from several distracters (e.g., a red square among green squares and red circles), target recognition becomes slower and error-prone. Even if the target itself is unique among the distracters, it shares at least one feature with any one of the distracters, which makes it less easily distinguishable. This task requires central attention and its difficulty increases proportionally with the number of distracters.

In yet other cases, target detection or recognition is facilitated by the presence of multiple target attributes. If a target object is defined by several features and the presence of either one of them on its own - as opposed to a combination of all target features - is sufficient to unambiguously recognize the target, target recognition is faster when more than one target feature is present. For example, red squares (i.e. targets with both features) will be detected faster than red circles or green squares (i.e. targets with only one of the two features). This is known as the Redundant Signals Effect (Kinchla, 1974) or the Redundant Target Effect (Miller, 1982).

The Redundant Target Effect (RTE) is a phenomenon that has proven to be consistent and stable whenever attention needs to be divided among several modalities, locations or feature dimensions, and when several input channels separately provide the necessary information to perform a task (Miller, 1982; Van der Heijden, La Heij \& Boer, 1983; Kinchla \& Collyer, 1974; Van der Heijden, 1975). Bimodal and even trimodal detection tasks show facilitation if a stimulus is presented on several different modalities more or less simultaneously (Bimodal: Wundt, 1880; Fidell, 1970; Mulligan \& Shaw, 1980; Miller, 1982; Trimodal: Van der Heijden et al., 1983; Diederich \& Colonius, 2004, Krummenacher, Müller \& Heller, 2001, Miller, 1981, Marzi et al., 1996).

The Redundant Target Effect generalizes across target dimensions - form, color, orientation, etc. (Miller, 1982; Mordkoff \& Yantis, 1993; Feintuch $\&$ Cohen, 2002), as well as letters and words (Morton, 1969) - and modalities (visual, auditory, and tactile; Diederich, 1995). The fact that reaction times profit from redundant signals, at least under most conditions and tasks, provides sound evidence that parallel rather than serial processing of input does happen. The RTE cannot be explained without assuming parallel processing at some stage of the processing pathway (Van der Heijden et al., 1983; Krummenacher et al., 2001; but see Townsend and Nozawa, 1995, who discuss a serial process that violate the Miller bound by assuming that the non-detection of an attribute is faster than its detection; Zehetleitner, Krummenacher, \& Müller, 2009, explored this issue).

However, several factors influence the size or the appearance of the RTE. Some of these are linked to the experimental design - for example non-target attribute presence or absence. If only one stimulus is present during single target trials, then the RTE is much smaller than if a distracter is present on the other channel during single target trials (Miller, 
1982; van der Heijden, Schreuder, Maris \& Neerincx, 1984). It seems that attention focused on one channel - as opposed to divided attention in cases where two or more channels have to be monitored - is sufficient to reduce, or in some cases, to completely compensate for any redundancy gain (Miller, 1982). The type of task is also important: redundancy gain is typically observed in experimental paradigms of the type Go-NoGo, where a response is required of the participant if, and only if any one of several redundant features is present (Miller, 1982; Mordkoff \& Yantis, 1991, 1993; Diederich 1995). Redundancy gain has also been found in a two-alternative-forced-choice paradigm (2AFC; Fidell, 1970), but it is not as large as in Go-NoGo paradigms (Grice \& Reed, 1992).

Spatial location of two redundant visual targets affects RTE as well: the farther they are apart, the lesser is the redundancy gain (Feintuch \& Cohen, 2002; Colonius \& Diederich, 2004). However, if targets in spatially different locations are bound together by grouping, the redundancy gain can be increased considerably, as they are then perceived as belonging to the same object (Feintuch \& Cohen, 2002).

Several types of models have been proposed to explain the redundant target effect: race models, coactivation models and various hybrids of these two. Both race and coactivation models are generally based on the assumption of independent channels that contribute to the accumulation of evidence. It is rather unlikely, however, that this assumption holds in reality. Mordkoff and Yantis (1991) showed that activity on one channel can be influenced by events on another channel, and several authors introduce lateral inhibition between channels to be able to explain their results on various reaction time tasks (Usher \& McClelland, 2001; Huber \& Cousineau, 2004).

The race model was one of the first models proposed to explain the RTE (Raab, 1962). It assumes independent channels separately accumulating evidence in favor of the specific signal or feature to which they are tuned. As soon as one of the channels has accumulated enough evidence to surpass the decision threshold, this channel - the fastest, hence the name of the model - determines the output of the model. The RTE is explained by the notion of statistical facilitation first introduced by Raab (1962). The author showed that when sampling random reaction times across channels, the distribution of the minimal response time of each of these samples will always have a mean lower than any of the response time distributions of the different channels. However, the starting point of the minimal response time distribution cannot be lower than the minimal response time across channels, but the variance of the minimal RT distribution will be smaller than the variance of any of the individual channel distributions.

Coactivation models were developed as an alternative to race models for explaining the RTE (Smith, 1968; Miller, 1982; Schwarz, 1989). Coactivation is defined as an activation build-up from different channels to satisfy a single threshold criterion. Coactivation models differ chiefly from race models in that the activation from the different channels is combined, at some point, in the processing of the input. Activation from all channels jointly determines what the response at the next processing level should be. In fact, it is the joint activation of a single threshold criterion from different channels which enables coactivation models to predict a redundancy gain: even if activation on any one channel alone is insufficient to overcome the threshold and make a decision, the pooling of activation from several still weakly activated channels makes it possible to overcome the threshold faster than with any single channel alone.

Various authors have compared separate activation and coactivation models (e.g., Mulligan \& Shaw, 1980; Fidell, 1970; Kinchla \& Collyer, 1974; Eriksen \& Schultz, 1979), but the conclusions are not homogeneous and are often contradictory (Mulligan \& Shaw, 1980; Fidell, 1970; Eriksen \& Schulz, 1979).

Several attempts have been made to find a criterion that allows a conclusive distinction between race models and coactivation models. A possible way of excluding separate activation models irrefutably was proposed by Miller (1978). The performance of race models on redundant target trials is 
simply the minimum response time of the different channels that contribute to the redundant signal (Raab, 1962). This allows us to calculate the best possible performance of race models. Two of the most well known methods to calculate the upper limit of race models are the Miller Inequality (Miller, 1978) and the Townsend Bound (Townsend \& Nozawa, 1995). If response time distributions on a redundant target task exceed either of these criteria, race models can be refuted as an explanation for redundancy gain; they are not capable of accounting for the amount of gain induced in redundant target trials.

The Miller Inequality has been used frequently to refute race models as the sole explanation for the redundancy gain in detection tasks with targets from different dimensions (Krummenacher, Müller \& Heller, 2002), different modalities (Diederich $\&$ Colonius, 1987), and letter search tasks (Miller, 1982). Even in participants with lateral visual extinction, the RTE induced by a stimulus in the extinct hemisphere was strong enough to violate the Miller Inequality (Marzi et al., 1996). In extending the Miller Inequality to include three redundant targets, Diederich and Colonius (2004) found evidence to refute race models in trimodal detection tasks: the gain observed between double redundant and triple redundant targets alone was too large to be explained solely by separate activation models.

Violation of the Miller Inequality, the Townsend Bound (see results) or any other criterion defining the upper limit of race model performance (e.g. Grice, Canham \& Gwynn, 1984) is usually interpreted as evidence of coactivation somewhere along the processing pathway. However, Mordkoff and Yantis (1991) suggested an alternative explanation: crosstalk. With the Interactive Race Model, they proposed an extension of separate activation models, which integrates inter-channel crosstalk (positive or negative contingencies between target and target, target and non-target, or two non-targets on two channels) and bias towards one response.

Mordkoff and Yantis (1991) explain Miller's (1982) and similar results in terms of existing contingencies between stimuli on different channels. In a series of experiments with a letter search task, they then show that the "Miller Inequality" is not violated if all contingencies between channels are equated.

In a rigorous test of the interactive race model (Schwarz, 1996), most of the results from Mordkoff and Yantis (1991) were replicated. However, under certain conditions (non-simultaneous signal presentation) violation of the "Miller Inequality" was consistently found even when inter-channel contingencies were equated. Miller (1981) also obtained violation of the Inequality in the absence of inter-channel correlations, as did Mordkoff and Yantis (1993). The latter concluded that although inter-channel crosstalk does influence response times, coactivation is at least partly responsible for facilitation in cross-dimensional redundant targets, whereas within a same dimension, separate activation is sufficient to explain facilitation.

This study pursues two different goals. First, we wish to investigate if redundancy gain from three redundant target attributes, inside a single modality, is possible. To the best of our knowledge, triple redundancy within any single modality has never been addressed before. One reason for expanding the study of redundancy gain in that direction is that the ecological validity of the target paradigm increases. In a natural context, we rarely see targets, which are defined by only two target attributes. Therefore, increasing the cognitive load and studying triple redundancy gain is likely to reveal interesting insights into processing of visual stimuli. Another reason for choosing a triple redundant paradigm is that it will likely give valuable information about the dynamics of a redundancy gain. It might give us an idea about an upper limit to gain in RTs, limits with respect to the type of target attributes, which can induce triple redundancy gain, and the factors that could hide or inhibit redundancy gain.

The second goal of this study is to differentiate between possible causes of redundancy gain. As mentioned above, the literature is not at all unified in attributing redundancy gain to statistical facilitation, to crosstalk or to coactivation. Based on our experimental data, we will exclude all three of these as an isolated explanation of the RTE. Since crosstalk is likely to exist, we exclude it 
by allowing no possible facilitatory contingencies between target attributes on different channels. In a second step, we will reject statistical facilitation by showing that performance of participants on redundant target recognition will be significantly above the Townsend Bound. In a final step, we will also refute coactivation models as an explanation for redundancy gain by comparing the minimal response time, as well as standard deviation and skew of participants' response time distribution with the coactivation model's predictions for those response time distribution characteristics.

\section{Method}

\section{Participants}

Participants were 24 undergraduate students (17 females) from the Université de Montréal, between 19 and 27 years of age. All had normal or corrected-to-normal vision. Participants were compensated with $8 \$$ per hour for their participation.

\section{Stimuli and Apparatus}

We used simple two-dimensional geometrical objects as stimuli. Stimuli were created in the RGB color space, using MatLab (MathWorks Inc., Natick, MA). Stimuli were presented using E-Prime (Psychology Software Tools, Inc., Pittsburgh, PA) on a SVGA monitor (refresh rate: 85 $\mathrm{Hz}$ ) at a distance of $80 \mathrm{~cm}$ from the participants. The stimuli measured 3 degrees of visual angle. They were either red, green, or blue; their form was a circle, a triangle or a square; and lastly they moved outward from the centre of the screen at an angle of 45 (right), 165 (down) or 285 (left) degrees. Stimuli were presented in front of an equiluminant gray background with stimulus luminance at 20 percent and stimulus saturation at 30 percent. Stimulus luminance and saturation was purposefully kept low to slow down color recognition. At full saturation, color is recognized much faster than form or direction of movement, and for the present purpose it was important to choose three attributes, which are recognized within roughly the same time frame.

Target stimuli possessed one or more of the following attributes: color blue, form of a square, and moving to the right. The presence of any single one of these attributes was sufficient to define a given stimulus as a target. Non-target stimuli did not possess any of the target attributes. They were green or red, a triangle or a circle and moving to the left or to the bottom of the screen.

\section{Design}

$50 \%$ of all stimuli presented to participants were targets. To avoid contingencies between attributes on different channels, which would facilitate redundant target recognition, the stimulus distribution shown in Table 1 was based on the three rules of contingency formulated by Mordkoff and Yantis (1991). However, this setup had three, instead of two feature channels, thus, exponentially more combinations of features that needed to be balanced. We could not control perfectly the contingencies but we did avoid the positive contingencies within target attributes, as well as between target and distracters attributes. This means that no facilitation of double and triple redundant targets due to existing contingencies could have occurred. However, inhibition of redundant stimulus recognition might be possible, since the non-target attributes on each channel do not occur as part of a target stimulus with the same frequency.

The second non-target attribute, which will be referred to as 'foil', is associated with non-target stimuli more frequently than the first. This was done to avoid facilitatory contingencies. Comparing foil and non-foil target-present trials gives us the means of testing the impact of negative contingencies (see results). Overall, none of the contingencies favored redundant target trials, reducing the chances of observing coactivation effects. 
Procedure

The experiment consisted of 16 blocks with 60 trials per block for a total of 960 trials. Eight of the participants participated in two sessions of the same experiment, performing 32 instead of 16 blocks, with a total of 1920 trials. Stimulus distribution did not vary between blocks, but the order of trials was randomized. Participants had the possibility to take a break between blocks, and were encouraged to do so, explicitly after eight blocks were completed.

The triple redundant target (target with all three target attributes present) was presented 48 times per participant. The six stimuli in double redundant conditions (any two target attributes present, plus one of two possible distracters on the third channel) were presented 48 times each. The three stimuli with only one target attribute were also presented 48 times each per participant. The fact that double-redundant trials were more frequent than the triple and singly redundant trials was necessary, in order to have a balanced stimulus distribution that avoided facilitatory contingencies (see above). Non-target stimuli were presented 480 times per participant.

Each trial started with the presentation of a blank screen for $494 \mathrm{~ms}$. The blank screen was followed by a fixation point for $694 \mathrm{~ms}$. The stimulus was then presented for a total of $823 \mathrm{~ms}$ during 9 frames, the first positioned at the screen center for $47 \mathrm{~ms}$. The subsequent 7 frames lasted $47 \mathrm{~ms}$, and gradually displaced the stimulus in the required direction of movement. The last frame lasted 447 ms, showing the stimulus at its final destination 3.78 degrees of visual angle from the screen center. Finally, a feedback slide appeared for $753 \mathrm{~ms}$.

For the reasons mentioned in the introduction, we used a Go-NoGo experimental paradigm. Participants were required to press the SPACE key on a keyboard as soon as they recognized a target stimulus, and discouraged from doing so if they recognized a non-target. They were encouraged to respond as fast as possible while making as few errors as possible. Responses had to happen within a time frame of 0 to $750 \mathrm{~ms}$ after stimulus onset.
Participants received feedback on their performance on each trial. Feedback on false responses was accompanied by a $700 \mathrm{~Hz}$ sound. Fast and correct performance was further encouraged by a system of points: participants were encouraged to try for the best score. Participants received 30 points for hits and correct rejections, 50 for particularly fast hits (under $300 \mathrm{~ms}$ ), and $\mathbf{- 3 5 0}$ points for false alarms and misses. At the end of each block, participants were given their cumulative score.

\section{Results}

The participants master the task very well, with an average of less than $1.4 \%$ of trials where they omitted to respond (13.03 of 960 trials per participant on average) and $2.5 \%$ false alarms (FA; 23.78 of 960 trials per participant on average). There was no significant difference between error rates for participants, which completed 16 or 32 blocks (Miss: $t(22)=-0.97, p=0.34$; FA: $t(22)=-1.09, p=0.29$ ). Neither was there a significant difference between mean response times for the two groups and seven conditions $(t(166)=0.16, p=0.87)$. Therefore, we may conclude that additional training had no significant effect. All further analysis will not take the different number of blocks into account.

Trials where participants responded faster than $205 \mathrm{~ms}$ were excluded from analysis, as these were considered anticipatory responses (a total of four trials were eliminated). For all further analysis, only correct Go-trials will be used. While maintaining a very high performance rate, participants also responded very rapidly: valid response times to a target could be as fast as $221 \mathrm{~ms}$. Participants maintained a mean response time of $398 \mathrm{~ms}$ across conditions, with a standard deviation (SD of 82 ms. Response times varied greatly, however, across conditions, and even more across participants. The mean response time for the triple redundant condition was $361 \mathrm{~ms}$ across participants, with a SD of $56 \mathrm{~ms}$. For double redundant conditions, the mean RT was slightly slower, at $389 \mathrm{~ms}$, with a SD of 74 ms. Finally, the mean response time for single-target conditions was $431 \mathrm{~ms}$ (SD: $93 \mathrm{~ms}$ ). 
TABLE 1.

Stimulus distribution for one block of 60 trials.

\begin{tabular}{|c|c|c|c|c|c|c|c|c|c|c|c|}
\hline \multicolumn{12}{|c|}{ Direction of motion } \\
\hline \multicolumn{4}{|c|}{ Right motion } & \multicolumn{4}{|c|}{ Left motion } & \multicolumn{4}{|c|}{ Down motion } \\
\hline & & Form & & & & Form & & & & Form & \\
\hline Color & square & circle & triangle & Color & square & circle & Triangle & Color & square & circle & triangle \\
\hline blue & 3 & 3 & 3 & blue & 3 & 3 & & Blue & 3 & & \\
\hline red & 3 & 3 & & red & 3 & & 3 & Red & & 3 & 3 \\
\hline green & 3 & & & green & & 3 & 3 & green & & 3 & 12 \\
\hline
\end{tabular}

Panels, rows and columns show the different attribute values. White fields are non-targets, light gray fields are stimuli with one target attribute, medium gray fields are stimuli with two target attributes, and the dark gray field represents a stimulus with all three-target attributes present.

\section{Difference in negative contingencies}

It is possible that performance was influenced by negative crosstalk due to the stimulus distribution (see Table 1). To empirically test this influence on participant performance, we compared error rates and mean response times in the double redundant conditions, splitting them according to the type of non-target attribute (foil or non-foil) they possessed. These conditions contained only one non-target attribute, allowing for a direct comparison. The double redundant conditions were those where the target possessed two target attributes, the color and the form (cf), the color and direction of movement (cd), or the form and direction of movement ( $\mathrm{fd}$ ).

Participants' error rates did not differ between types of non-target for any of the three double redundant conditions (cf: $t(46)=-0.23, p=0.41 ; \mathrm{cd}: t$ $(46)=-0.91, p=0.18 ; \mathrm{fd}: t(46)=-0.56, p=0.29)$. The same holds true for mean response times: they did not differ significantly between types of non-targets for any of the double redundant conditions (cf: $t$ (46) $=-0.65, p=0.26$; $\mathrm{cd}: t(46)=-1.66, p=0.052 ; \mathrm{fd}:$ $t(46)=-0.26, p=0.4)$. We can therefore conclude that negative contingencies do not affect response times, at least in the double redundant conditions. Hereafter, no distinction will be made between different non-target types in the double redundant conditions.

\section{Redundancy gain}

Figure 1 presents the mean response times to a target in conditions where a single target attribute was presented (color only: c, form only: f, or direction of motion only: $d$ ), in conditions where two target attributes were presented (conditions $\mathrm{cf}, \mathrm{cd}$, or $\mathrm{fd}$ ) and where all three target attributes were presented (condition cfd). As can be seen in Figure 1, there are benefits of redundant targets at the level of the mean RT, both for double redundant (2Red) over single-attribute conditions (1Red) and for triple redundant (3Red), over double redundant conditions.

Figure 2 shows the cumulative response time distributions of one representative participant, for all three single-target conditions, as well as for all three conditions where two target attributes were presented simultaneously. The probability of responding at time $t$ or faster is plotted as a function of time.

Due to the large inter-participant differences in overall response time distributions, systematic changes in response times depending on condition are hard to see if analysis is done over all participants. Therefore, to test for redundancy gain, we do not test for statistically significant differences between distributions with multiple t-tests, as did Miller (1982), as well as Mordkoff and Yantis (1991, 1993), among others. ${ }^{1}$ Instead, we assessed the dif-

1 When we do, we find, congruent with the individual analyses reported next that the differences are significant at the .05 level for quantiles 0.25 to 0.35 in the cf condition, for quantile 0.25 to 0.55 in the cd condition, and for quantiles 0.30 to 0.45 in the 
ference between two cumulative distributions of response times for different conditions from one

fd condition (quantiles went from 0.05 to 0.95 by increment of $0.05)$. In the triple redundant condition, the Miller bound is inadequate and therefore, we use the Diederich bound (Diederich, 1992). We find significant results for quantiles 0.35 to 0.80 and 0.90 , most of which are significant at the 0.01 level.

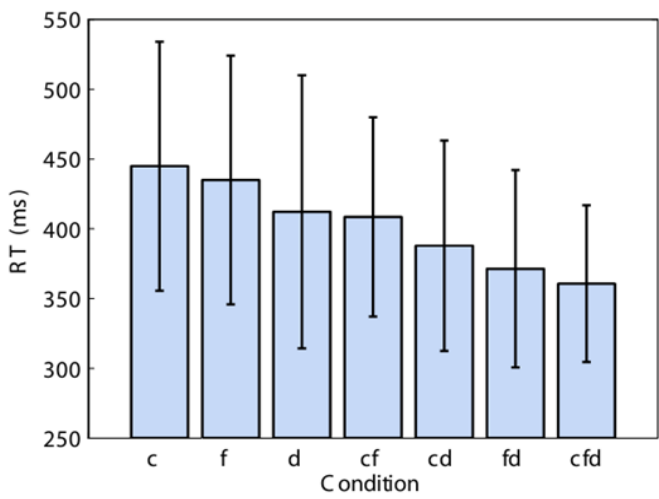

Figure 1. Mean response time per condition over all participants. Error bars show standard deviation. The abbreviation c stands for color, $\mathrm{f}$ for form, and $\mathrm{d}$ for direction of movement. The combination cf stands for stimulus containing both the target color and the target form.

Source: Own work. participant using a two-sample Anderson-Darling test of cumulative distributions (Anderson \& Darling, 1952) at a level of significance of 0.05. This test was chosen as it is more sensitive to differences at the extreme ends of distributions, than the wellknown Kolmogorov-Smirnoff test (Engmann \& Cousineau, 2011).

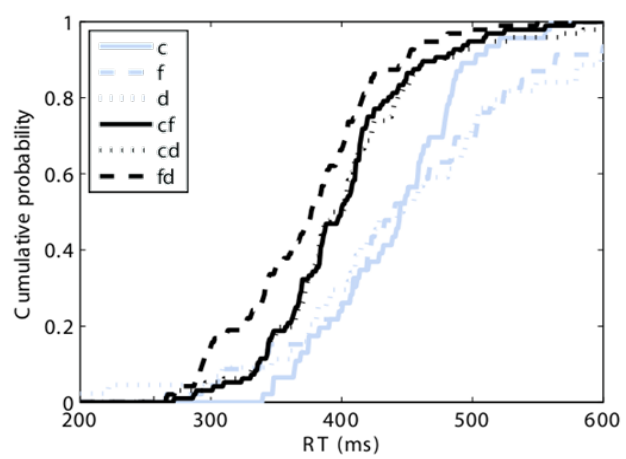

Figure 2. RT distributions of participant 13 for single- and double redundant conditions. Gray lines are the cumulative distributions for single-target conditions, black lines for double redundant conditions. All double redundant conditions for this participant are significantly faster than the non-redundant conditions. The abbreviations are the same as in Figure 1. Source: Own work.

TABLE 2

Proportion of participants for whom the distribution in a condition was significantly different from the distribution in a condition with less redundancy.

\begin{tabular}{cccc}
\hline $\begin{array}{c}\text { Double Redundant } \\
\text { Conditions }\end{array}$ & $\mathrm{c}$ & Non-redundant conditions \\
\hline $\mathrm{cf}$ & $21 / 24$ & $\mathrm{f}$ & $\mathrm{d}$ \\
$\mathrm{cd}$ & $(11.29 / 1.56)^{*}$ & $(7.52 / 0.99)^{*}$ & - \\
$\mathrm{cd}$ & $24 / 24$ & - & $15 / 24$ \\
$\mathrm{fd}$ & $(19.92 / \text { n.a. })^{* *}$ & $23 / 24$ & $(6.36 / 1.41)^{*}$ \\
& & $(23.28 / 1.85)^{*}$ & $(11.36 . / 1.33)^{*}$ \\
\hline & & & \\
Triple & & Double redundant conditions & $\mathrm{fd}$ \\
Redundant Condition & $\mathrm{cf}$ & $\mathrm{cd}$ & $7 / 24$ \\
$\mathrm{cfd}$ & $24 / 24$ & $18 / 24$ & $(5.9 / 1.2)^{*}$ \\
\hline
\end{tabular}

* The critical AD for a decision criterion of 0.05 is 2.49 .

** For one participant, there was a significant difference between the RT distributions for c and cd, but in the opposite direction; i.e. the RT for $\mathrm{c}$ was actually faster than for $\mathrm{cd}$. However, this was the only case where such a reversal was observed.

Between parentheses, the mean Anderson-Darling statistics (AD) for the participants with a significant difference, as well as for participants with a non-significant difference. The abbreviation $\mathrm{c}$ stands for color, $\mathrm{f}$ for form, and $\mathrm{d}$ for direction of movement. The combination cf stands for stimulus containing both the target color and the target form.

Source: Own work 


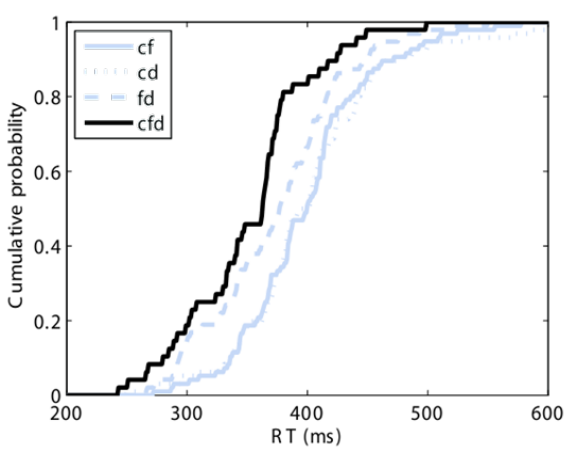

Response time distributions to double redundant stimuli with color and form present were significantly faster than RTs for color only for almost all participants (21 out of 24 ), and significantly faster than the RTs for form only (17/24 participants). The same holds for double redundant stimuli with color and direction of movement present (24/24 and $15 / 24$ participants respectively) and for form and direction of movement (23/24 and 20/24 participants respectively). The upper part of Table 2 recapitulates the results.

Figure 3 shows the cumulative response time distributions for a representative participant of the three double redundant conditions, as well as when all three target attributes are present. Since participants' reaction times were already very fast for the double redundant conditions, an increase in RT becomes more difficult to observe for triple redundant targets. Therefore, even though a general trend was visible, and most participants responded faster for triple redundant stimuli than for any double redundant stimuli, the difference between $\mathrm{fd}$ and $\mathrm{cfd}$ conditions was significant for only 7 participants (fd: $7 / 24$ participants). However, in the other two conditions (color $\&$ direction of movement, cd and color $\&$ form, cf), the proportion of participants that have a significantly faster distribution in the triple redundant condition is greater (cd: 18/24; cf: 24/24). Table 2, bottom part, gives the details for all the double redundant target distributions relative to the triple redundant target condition.

These results show that it is indeed possible to profit from three redundant attributes for object recognition, even though the benefit of a third attribute is not always significant across participants.
Figure 3. RT distributions of participant 13 for double and triple redundant conditions. Gray lines are the cumulative distributions for double redundant conditions, the black line for the triple redundant condition. This participant is significantly faster in the triple redundant condition than in any of the double redundant conditions. The abbreviations are the same as in Figure 1. Source: Own work.

\section{Excluding Race Models}

After having found that redundancy gain exists for three redundant attributes within the same modality, we now need to distinguish between the possible causes of this gain. Since facilitation by crosstalk was excluded a priori (see method), this leaves coactivation and statistical facilitation as possible explanations. Since statistical facilitation has an upper limit to the amount of gain it can explain (see introduction), we will use this upper limit to differentiate between these two causes of gain.

The Miller Inequality is commonly used, as it gives a definite upper limit to the performance of race models with two channels, and is therefore a very efficient criterion of exclusion for race models on any task with two redundant targets. However, one cannot estimate the degree of dependence between channels, as it is not directly observable. Hence a generalization of the Miller Inequality to three or more channels poses a problem: the degree of dependence between any two channels needs to be subtracted from the sum of the response time distributions of the separate channels, but then, the degree of dependence of the three channels would have to be added again. Both values are positive but of unknown size, making it impossible to determine in which direction the extension of the Miller Inequality would tend.

An alternative to the Miller Inequality was proposed by Townsend (Townsend \& Ashby, 1983; Townsend \& Nozawa, 1995, among others; a similar bound was proposed by Mordkoff \& Yantis, 1991, p. 535; as well as by Luce, 1986, p. 162). It is based upon survivor functions (one minus the cumulative distribution) of response times, instead of cumulative distribution functions. The upper limit to race 
model performance with more than one channel is given by the survivor function of the product of the survivor functions of each channel:

$\operatorname{Pr}\left(\mathbf{R T}_{c d f}<t\right)=1-\prod_{i \in\{c, d, f\}} 1-\operatorname{Pr}\left(\mathbf{R} \mathbf{T}_{i}<t\right)(1)$ where $R T_{\text {cfd }}$ is a response time in the triple redundant condition and $i$ indexes the three conditions with only one target attribute. If the observed response time distribution in a redundant target task is significantly faster than predicted by this boundary, race models as the sole explanation of redundancy gain are rejected. The Townsend Bound can be calculated for any number of channels. We again used the AD test to test for significant differences between the redundant target distribution and the Townsend Bound calculated from the corresponding single-target RT distributions.

Figure 4 shows the single-target distributions of a representative participant, as well as the Townsend Bounds and the actual response time distributions for each of the three double redundant conditions. The AD test compares the dotted line to the full line in this figure.

For double redundant targets with color and form present, the RT distributions were significantly faster than the Townsend Bound calculated from the RT distributions of single-target color-only and form-only targets for two participants only $(A D=$ 2.87 ; 4.23). For color and direction of movement, four participants' RT distributions were significantly faster than the corresponding Townsend Bound ( $\mathrm{AD}=3.09 ; 3.43 ; 3.68 ; 5.09)$; and for form and direction of movement, twelve participants' RT distributions were significantly faster than the corresponding Townsend Bound $(\mathrm{AD}=7.18 ; 4.92$; $4.39 ; 5.52 ; 7.07 ; 10.03 ; 2.99 ; 12.43 ; 5.16 ; 37.25$; $2.85 ; 8.44)$.

To test for coactivation in the triple redundant target condition, the Townsend Bound was calculated from all three single-target RT distributions. Figure 5 shows the three single-target distributions of a representative participant, the Townsend Bound and the actual distribution for the triple redundant condition. Eleven out of 24 participants performed significantly faster than predicted by the Townsend Bound on triple redundant target trials $(\mathrm{AD}=5.42$; $4.64 ; 6.43 ; 4.23 ; 3.81 ; 3.34 ; 8.56 ; 13.25 ; 2.50 ; 5.13$; 6.89).

\section{Excluding race models for gain from}

double to triple redundancy

The Townsend Bound for triple redundant targets, as formulated above, is based on the RT distributions of single-target trials. It is able to assert that the interaction between three single target attributes is more than statistical facilitation. This opens the possibility that two attributes only coact-
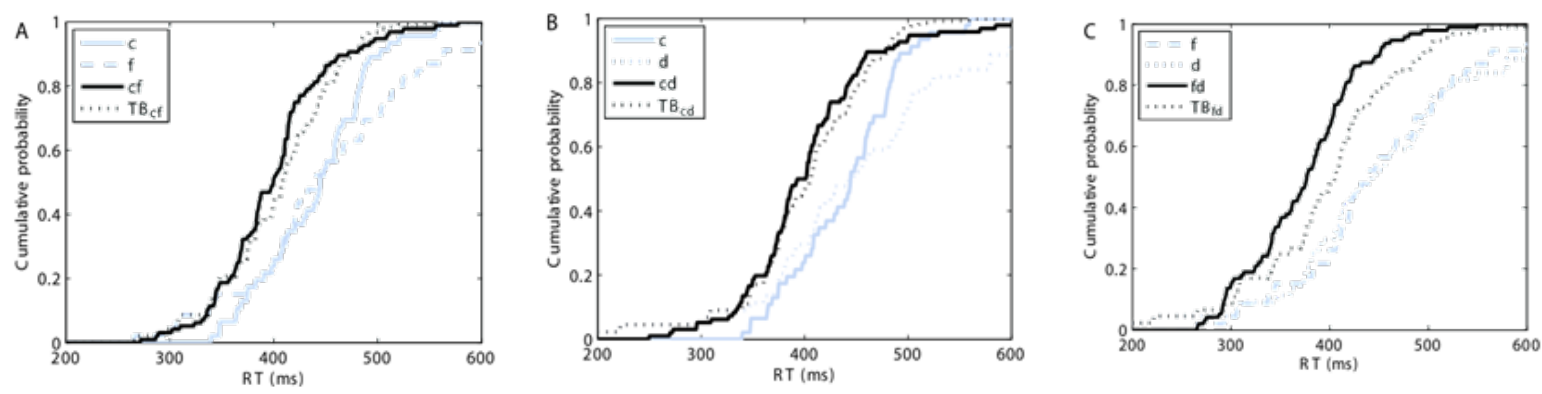

Figure 4. Townsend Bound and RT distributions of participant 13 for double redundant conditions. Panels represent the three different combinations of two target attributes. Gray lines are the cumulative distributions for single-target conditions, the continued black line the respective double redundant condition and the dotted black line shows the corresponding Townsend Bound (TB). This participant responds significantly faster than the corresponding Townsend Bound only for the condition fd (see panel C). In the other two conditions (Panels A and B) there is no significant difference between the Townsend Bound and actual RT distribution. The abbreviations are the same as in Figure 1.

Source: Own work. 


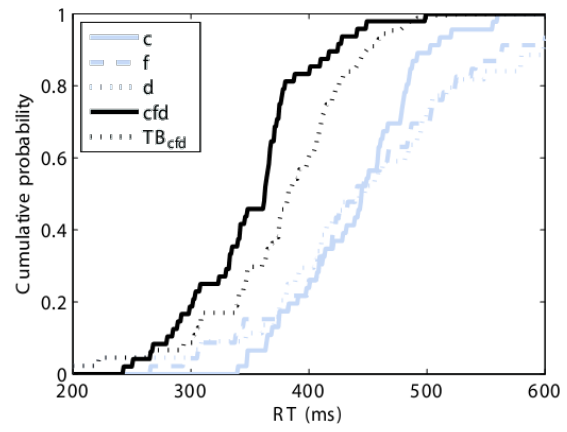

ivate, and the third one contributes only within the range of statistical facilitation. In order to show that statistical facilitation cannot be responsible for the amount of gain obtained from an additional third attribute, we need to adapt the Townsend Bound. We can calculate the Townsend Bound for triple redundancy for the RT distribution of any of the three double redundant conditions, plus the RT distribution of the respectively missing single-target condition. We then obtain a new bound by taking the maximum of the three above-mentioned values at each time point.

$$
\begin{aligned}
& \operatorname{Pr}\left(\mathbf{R T}_{c d f}<\mathrm{t}\right)=\max \left[1-\left(1 \operatorname { P r } ( \mathbf { R T } _ { \text { cf } } < \mathrm { t } ) \mathbf { x } \left(1-\left(\operatorname{Pr}\left(\mathbf{R T}_{d}<\mathrm{T}\right)\right),\right.\right.\right. \\
& 1-\left(1 \operatorname{Pr}\left(\mathbf{R T}_{c d}<t\right) \times\left(1-\left(\operatorname{Pr}\left(\mathbf{R T}_{f}<\mathrm{T}\right)\right),(2)\right.\right. \\
& 1-\left(1 \operatorname{Pr}\left(\mathbf{R T}_{f d}<t\right) \times\left(1-\left(\operatorname{Pr}\left(\mathbf{R T}_{c}<T\right)\right)\right. \text {, }\right.
\end{aligned}
$$

The maximum returns the most conservative evaluation of performance if one of the three target attributes contributes only by statistical facilitation as a third redundant attribute. For example, if only the two attributes $c$ and $f$ have coactivation effects (or any other form of dependencies that makes RTs faster, then the top line in Eq. 2 will be selected by the max function (the CDF of the pair $c f$ will grow faster). Hence, exceeding this limit can only be achieved if all three target attributes contribute above statistical facilitation to the amount of redundancy gain. Equation 2 was first derived in Engmann (2009). The appendix reviews the existing bounds and explains in what way Eq. 2 is a conservative bound relative to other bounds.

The above bound for triple over double redundancy could have been calculated from the product of the survivor functions of the three double redundant conditions, analogous to equation (1). However, in this case, the gain contributed by each
Figure 5. Townsend Bound and RT distributions of participant 13 for triple redundant conditions. Gray lines are the cumulative distributions for single-target conditions, the continued black line the triple redundant condition and the dotted black line shows the corresponding Townsend Bound (TB). The participant is significantly faster in the triple redundant condition than predicted by the corresponding Townsend Bound. The abbreviations are the same as in Figure 1.

Source: Own work.

attribute would be included twice (once in each of the two double redundant conditions it is part of), thereby obtaining an upper limit, which would definitely exceed performance of a combination of coactivation for two and statistical facilitation for the third attribute. Equation (2) ensures that each attribute contributes only once, while still ensuring the best possible performance under the assumption that statistical facilitation is responsible for the gain attributed to the third target attribute.

Two participants performed significantly faster on triple redundant trials than predicted by the triple over double redundancy Engmann Bound $(A D=2.9 ; 2.5)$. Figure 6 shows the cumulative RT distributions for double redundant and triple redundant conditions for each of these participants respectively, as well as the Townsend Bound obtained from the non-redundant conditions (Eq. 1) and the Engmann Bound obtained from the double redundant conditions (Eq. 2). This shows that gain, solely from a third redundant attribute, cannot be accounted for by statistical facilitation. We can, therefore, conclude that all three target attributes must interact in some other way than statistical facilitation to contribute to a triple redundancy gain.

In conclusion, we show that redundancy gain from a third attribute is indeed possible, even though all attributes come from the same modality, and despite the fact that performance for double redundant targets is already very fast.

The theoretical bound of Eq. 2 is not violated for every participant and every condition. This can have several possible causes. First, since participants responded very rapidly, even to single-target conditions, room for improvement under redundant conditions is limited. This is supported by the fact 
that 18 of 24 participants' triple redundant distributions tended to be above the Engmann Bound, even if the AD test was not significant for 13 of the 24 participants.

Second, the potential for improvement under redundant conditions is maximal if single attributes are processed at approximately the same rate. If one attribute is visibly slower than another, the advantage of adding this target attribute is smaller than if attributes are processed at the same speed. ${ }^{2}$ As can be seen in Figure 1, the attribute color may be slightly slower than the attribute direction of movement. The argument that attributes need to be processed at approximately the same speed to be able to observe maximal triple redundancy gain, is supported by the results of this study: Redundancy gain was found for those participants that did indeed process all three single attributes at the same speed, and more specifically also processed double redundant targets at roughly the same speed.

Third, to exclude crosstalk as the source of the RTE for the triple redundant target, contingencies between attributes were permitted. More specifically, there exists a positive relation between the presence of a target attribute and a non-target attribute.

2 The choice of attributes for this study was made based on pilot experiments testing the overall reaction times to a series of attributes. From among orientation, spatial frequency, size, letters, direction of movement, color, and form - with varying degrees of saturation and luminosity to modify processing speed of certain attributes - the latter three proved to elicit approximately the same reaction times for most participants, under conditions of low luminosity and saturation.

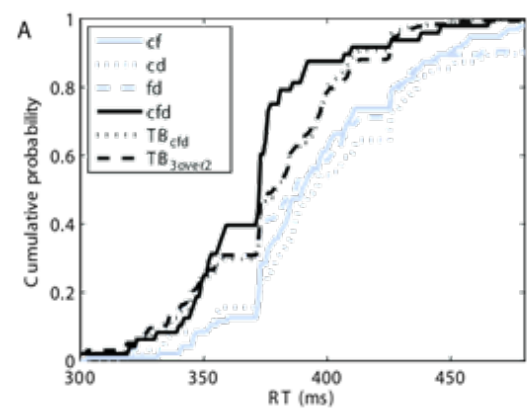

However, there is an opposite relation between the presence of a pair of target attributes and the presence of a pair of target or non-target attributes. Hence, the former might inflate the double-redundant effect, but triple redundant effect owes nothing to these contingencies. Since the possibility that crosstalk exists cannot be excluded, the bound presented in Equation 3, using the double-redundant condition, might be even more conservative than we wished for. Note, however, that we checked that the double-redundant contingency effect is not important (comparing non-target and foil stimuli).

Lastly, gain from redundant target conditions can be outweighed by sources of noise or inhibition. Since there is already very little room for improvement from double to triple redundancy, it would not take much to mask a triple RTE. Although there are several possible internal sources of noise, one should be mentioned specifically. We excluded crosstalk as an explanation of redundancy gain, by avoiding contingencies that facilitate redundant object recognition. We could not however avoid inhibitory contingencies. If we assume that crosstalk between channels exist, it would work against a gain from redundancy under these experimental conditions. However, our analysis of the impact of foils versus non-targets (see above) seems to indicate that inhibitory contingencies had no or only a negligible impact on the data.

Nevertheless, the violations we did obtain are enough to refute statistical facilitation as an incomplete explanation of redundancy gain. Since we

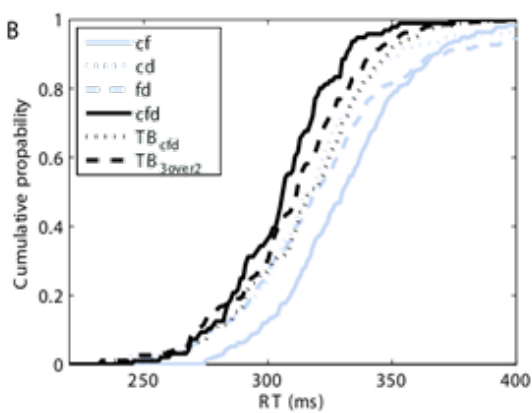

Figure 6. Townsend Bound and Townsend Bound calculated from double redundant distributions for participants 25 (panel A) and 39 (Panel B). Gray lines are the cumulative distributions for double redundant conditions; the continued black line the triple redundant condition, the dotted black line shows the corresponding Townsend Bound (TBcfd), and the dashed black line the corresponding Engmann Bound calculated from double redundant conditions (TB3over2, Eq. 2.). Both participants' RTs are significantly faster in the triple redundant condition than predicted by both bounds. The abbreviations are the same as in Figure 1.

Source: Own work. 
excluded crosstalk as a possible explanation by the specific experimental design we used, this leaves us with only coactivation as an option for explaining the size of the redundancy gain observed under these conditions.

\section{Discussion}

This study is the first to find a triple redundancy gain from attributes within a single modality. Not all participants showed a triple redundancy gain, and for those that did, not all overcame the threshold given by the Engmann Bound. This, as well as the lack of other studies with similar findings, is caused by the difficulty of creating the right conditions under which such a gain might be observed (as discussed previously). Despite these difficulties, the current results allow an important conclusion. Finding a triple redundancy gain within a single modality that violates the Townsend Bound is possible, and separate activation race models cannot explain it.

There has been an extensive discussion concerning the stage along the processing pathway, which is responsible for violating the Miller Inequality. Since this violation was mainly taken as evidence for coactivation, various types of coactivation models have been proposed, with coactivation happening at different stages of the processing pathway. Fidell (1970) and Miller (1982) both assume coactivation takes place at a very early stage of processing, the detection level (activation from both channels is pooled in order to detect a signal in either modality). However, other variants of coactivation models have been proposed. The Logogen model (Morton, 1969) predicts performance on bimodal word recognition tasks, and postulates coactivation during the recognition process (activation from all channels that have signaled the detection of a signal is pooled to enable identification).

Keele (1973) extended the Logogen model to include coactivation at the decision level (signals on different channels are recognized separately but feed into a common pool to decide the appropriate response). A model proposed by Logan (1980) predicts performance during response competition (e.g., the Stroop task), also with coactivation at the decision level. A model of visual search with coactivation at the decision level has also been proposed (Eriksen \& Schulz, 1979). But even if all processing up to the decision level is based upon separate activation, response times can still profit from coactivation at the response initiation level or even at the motor stage. Input even from unmonitored modalities increases the general state of arousal, thereby facilitating response initiation (Nickerson, 1973). Differences in reaction time between responses with the right or left hand (only right hand responses showed enough redundancy gain for coactivation) indicate that at least part of the facilitation for redundant targets arises at a motor level (Diederich \& Colonius, 1987).

In conclusion, the results of this study show that redundancy gains from a third attribute within one modality is possible, that this redundancy gain is not compatible with separate activation models. However, even if these results are compelling for coactivation models, we note that they do not represent evidence in favor of coactivation unless we could show that there is no alternative model. We should, therefore, work at deriving predictions from the coactivation model and see if they are falsified.

\section{Acknowledgements}

The authors would like to thank Dominic Charbonneau and Laurence Richard for their help and Sophie Callies, Étienne Dumesnil, Laurence Morissette and two anonymous reviewers for comments on a previous version of the manuscript.

\section{References}

Anderson, T. W., \& Darling, D. A. (1952). Asymptotic theory of certain goodness-of-fit criteria based on stochastic processes. Annals of Mathematical Statistics, 23, 193-212.

Colonius, H., \& Diederich, A. (2004). Multisensory Interaction in Saccadic Reaction Time: A Time-Window-of-Integration Model. Journal of Cognitive Neuroscience, 16(6), 1000-1009.

Diederich, A. (1992). Probability inequalities for testing separate activation models of divided attention. Perception and Psychophysics, 52, 714-716. 
Diederich, A. (1995). Intersensory facilitation of reaction time: Evaluation of counter and diffusion coactivation models. Journal of Mathematical Psychology 39, 197-215.

Diederich, A., \& Colonius, H. (1987). Intersensory facilitation in the motor component? A reaction time analysis. Psychological Research, 49, 23-29.

Diederich, A., \& Colonius, H. (2004). Bimodal and trimodal multisensory enhancement of reaction time: Effects of stimulus onset and intensity. Perception and Psychophysics, 66, 1388-1404.

Engmann, S. (2009). Redundancy gain: manifestations, causes, and predictions. Université de Montréal: unpublished doctoral dissertation.

Engmann, S., \& Cousineau, D. (2011). Comparing distributions: the two-sample Anderson-Darling test as an alternative to the Kolmogorov-Smirnoff test. Journal of Applied Quantitative Methods, 6, 1-17.

Eriksen, C., \& Schulz, D. (1979). Information processing in visual search: A continuous flow conception and experimental results. Perception and Psychophysics, 25, 249-263.

Feintuch, U., \& Cohen, A. (2002). Visual attention and co-activation of response decisions for features from different dimensions. Psychological Science, $13,361-369$.

Fidell, S. (1970). Sensory Function in Multimodal Signal Detection. Journal of the Acoustical Society of America, 47(4), 1009-1015.

Gnedenko, B. (1943). Sur la distribution limite du terme maximum d'une série aléatoire. Annals of Mathematics, 44, 423-453.

Grice, G. R., Canham, L., \& Gwynne, J. W. (1984). Absence of redundant-signals effect in a reaction time task with divided attention. Perception and Psychophysics, 36, 565-570.

Grice, G. R., \& Reed, J. M. (1992). What makes targets redundant? Perception and Psychophysics, 51(5), $437-442$.

Gumbel, E. J. (1958). The Statistics of Extremes. New York: Columbia University Press.

Heathcote, A., Brown, S., \& Cousineau, D. (2004). QMPE: Estimating Lognormal, Wald and Weibull RT distributions with a parameter dependent lower bound. Behavior Research Methods, Instruments, $\mathbb{E}$ Computers, 36, 277-290.
Hélie, S. (2006). An introduction to model selections: Tools and algorithms. Tutorials in Quantitative Methods for Psychology, 2, 1-10.

Huber, D. E., \& Cousineau, D. (2004). A race model of perceptual forced choice reaction time. Proceedings of the 25th Annual Meeting of the Cognitive Science Society, Boston.

Kandel, E. R., Schwartz, J. H., \& Jessell, T. M. (2000). Principles of Neural Science. New York: McGraw-Hill.

Keele, S. (1973). Attention and human performance. Pacific Palisades, CA: Goodyear.

Kinchla, R. A., \& Collyer, C. E. (1974). Detecting a target letter in briefly presented arrays: A confidence rating analysis in terms of a weighted additive effects model. Perception and Psychophysics, 16(1), 117-122.

Krummenacher, J., Müller, H. J., \& Heller, D. (2001). Visual search for dimensionally redundant pop-out targets: Evidence for parallel-coactive processing of dimensions. Perception and Psychophysics, 63, 901-917.

Krummenacher, J., Müller, H. J., \& Heller, D. (2002). Visual Search for Dimensionally Redundant Pop-Out Targets: Parallel-Coactive Processing of Dimensions Is Location Specific. Journal of Experimental Psychology: Human Perception and Performance, 28(6), 1303-1322.

Logan, G. D. (1980). Attention and automaticity in Stroop and priming tasks: Theory and data. Cognitive Psychology, 12, 523-553.

Logan, G. D. (1992). Shapes of reaction-time distributions and shapes of learning curves: a test of the instance theory of automaticity. Journal of Experimental Psychology: Learning, Memory and Cognition, $18,883-914$.

Loftus, G. R., \& Masson, M. E. J. (1994). Using confidence intervals in within-subject designs. Psychonomic Bulletin E⿱乛 Review, 1, 476-490.

Luce, R. D. (1986). Uniqueness and Homogeneity of Ordered Relational Structures. Journal of Mathematical Psychology, 30, 391-415.

Marzi, C. A., Smania, N., Martini, M. C., Gambina, G., Tomelleri, G., Palamara, A., Alessandrini, F., \& Prior, M. (1996). Implicit redundant-target effects in visual extinction. Neuropsychologia, 34(1), 9-22.

McClelland, J. L. (1979). On the time relations of mental processes: An examination of systems of processes in cascade. Psychological Review, 86, 287-330. 
Miller, J. (1978). Multidimensional same-different judgments: Evidence against independent comparison of dimensions. Journal of Experimental Psychology: Human Perception and Performance, 4, 411-422.

Miller, J. (1981). Global precedence in attention and decision. Journal of Experimental Psychology: Human Perception and Performance, 9, 1161-1174.

Miller, J. (1982). Divided attention: Evidence for coactivation with redundant signals. Cognitive Psychology, 14, 247-279.

Miller, J., \& Ulrich, R. (2003). Simple reaction time and statistical facilitation: A parallel grains model. Cognitive Psychology, 46, 101-151.

Milner, A. D., \& Goodale, M. A. (1993). Visual pathways to perception and action. Progress in Brain Research, 95, 317-337.

Morey, R. D. (2007). Confidence Intervals from Normalized Data: A correction to Cousineau (2005). Tutorial in Quantitative Methods for Psychology, 4(2), 61-64.

Mordkoff, J. T., \& Yantis, S. (1991). An interactive race model of divided attention. Journal of Experimental Psychology: Human Perception and Performance, 17, 520-538

Mordkoff, J. T., \& Yantis, S. (1993). Dividing attention between color and shape: Evidence of co-activation. Perception and Psychophysics, 53, 357-366.

Morton, J. (1969). Interaction of information in word recognition. Psychological Review, 76, 165-178.

Mulligan, R. M., \& Shaw, M. L. (1980). Multimodal signal detection: Independent decision vs. integration. Perception and Psychophysics, 28, 471-478.

Nickerson, R. S. (1973). Intersensory facilitation of reaction time: Energy summation or preparation enhancement? Psychological Review, 80(6), 489-509.

Raab, D. H. (1962). Statistical facilitation of simple reaction times. Transactions of the New York Academy of Sciences, 24(5), 574-590.

Schwarz, W. (1989). A new model to explain the redundant-signal effect. Perception and Psychophysics, 46(5), 498-500.

Smith, E. E. (1968). Choice reaction time: An analysis of the major theoretical positions. Psychological Bulletin, 69, 77-110.
Townsend, J. T. \& Ashby, F. G. (1983). Stochastic Modeling of Elementary Psychological Processes. Cambridge, England: Cambridge University Press.

Townsend, J. T., \& Nozawa, G. (1995). Spatio-temporal Properties of Elementary Perception: An Investigation of Parallel, Serial, and Coactive Theories. Journal of Mathematical Psychology, 39, 321-359.

Treisman, A., \& Souther, J. (1985). Search asymmetry: a diagnostic for preattentive processing of separable features. Journal of Experimental Psychology: General, 114(3), 285-310.

Tremblay, L.-M. \& Chassé, Y. (1970). Introduction à la Méthode Expérimentale, Montréal: Centre Educatif et Culturel inc.

Ulrich, R., \& Miller, J. (1993). Information processing models generating lognormally distributed reaction times. Journal of Mathematical Psychology, 37, 513-525.

Ungerleider, L. G., \& Mishkin, M. (1982). Two Cortical Visual Systems. In D. J. Ingle, M. A. Goodale, \& Mansfield, R. J. W. (Eds.). Analysis of Visual Behavior (pp. 549-586). Cambridge, MA: MIT Press.

Usher, M., \& McClelland, J. L. (2001). The time course of perceptual choice: The leaky, competing accumulator model. Psychological Review, 108, 550-592.

Van der Heijden, A. H. C. (1975). Some evidence for a limited capacity parallel self terminating process in simple visual search tasks. Acta Psychologica, 39(1), 21-41.

Van der Heijden, A. H. C., La Heij, W., \& Boer, J. P. A. (1983). Parallel Processing of Redundant Targets in Simple Visual Search Tasks. Psychological Research, $45,235-254$.

Van der Heijden, A. H. C., Schreuder, R., Maris, L., \& Neerincx, M. (1984). Some evidence for correlated separate activation in a simple letter-detection task. Perception and Psychophysics, 36, 577-585.

Wundt, W. (1880). Grundzüge der physiologischen Psychologie. (2nd ed.). Leipzig: Engelmann.

Zehetleitner, M., Krummenacher, J., \& Müller, H. J. (2009). The detection of feature singletons defined in two dimensions is based on salience summation rather than on serial exhaustive or interactive race architectures. Attention, Perception $\mathbb{E}$ Psychophysics, $71,1739-1759$ 


\section{Appendix: Coactivation bounds}

Review of the bounds

A certain number of bounds have been proposed to discriminate from statistical facilitation. The most famous is the Miller inequality (1982):

$F_{a b}(t) \leq F_{a}(t)+F_{b}(t)[$ Miller $]$

where $F_{\text {channels }}(t)$ is a shortcut for $\operatorname{Pr}\left(\mathrm{RT}_{\text {channels }}<t\right)$. The Miller inequality can be derived from a Venn diagram. By ignoring the intersection of $a$ and $b$, we get the inequality. Grice and his colleagues (1984) did not ignore the intersection, but assuming independence of channel processing, got the following equality $F_{a b}(t)=F_{a}(t)+F_{b}(t)-F_{a}(t) \times F_{b}(t)$, which can be factorized into $F_{a b}(t)=1-\left(1-F_{a}(t)\right)\left(1-F_{b}(t)\right)$. This last expression was already known for a long time (e.g., Townsend and Ashby, 1983) and has the advantage of being generalizable to any number of independent channels, so that:

$F_{a b c}(t)=1-\prod_{i \in\{a, b, c\}}\left(1-F_{i}(t)\right)[$ Townsend]

The Miller bound cannot be extended to three processing channels in any obvious ways. Diederich proposed an extension, so that:

$$
\begin{array}{r}
F_{a b c}(t) \leq \operatorname{Min}\left[F_{a b}(t)+F_{a c}(t)-F_{a}(t),\right. \\
F_{a b}(t)+F_{b c}(t)-F_{b}(t), \\
\left.F_{a c}(t)+F_{b c}(t)-F_{c}(t)\right]
\end{array}
$$

[Diederich]

This bound has the advantage of allowing possible dependencies (pair-wise or triple-wise). However, it uses a min rule which makes this bound a liberal bound, as shown in the next section.

The Townsend bound likewise can be extended by noting that

$$
1-\Pi_{i \in\{a, b, c\}}\left(1-F_{i}(t)\right)=1-\prod_{i \in\{a, b\}}\left(1-F_{i}(t)\right) \times\left(1-F_{c}(t)\right)
$$

With this formulation, we can mix observed performance in double-redundant conditions with performance in no redundancy conditions. If there exist one pair of channels that coactivate, or that are dependent in a favorable way, this pair is surely included in the bound by using a max rule. Hence, we get this new bound, first proposed in Engmann, 2009:

$$
\begin{aligned}
F_{a b c}(t) \leq \operatorname{Min}\left[1-\left(1-F_{a b}(t)\right) \times\left(1-F_{c}(t)\right),\right. \\
1-\left(1-F_{a c}(t)\right) \times\left(1-F_{b}(t)\right), \\
\left.1-\left(1-F_{b c}(t)\right) \times\left(1-F_{a}(t)\right)\right]
\end{aligned}
$$

[Engmann]

This equation is presented in the text as Equation 2.

\section{Liberal and conservative bounds}

Both Diederich and Engmann bounds are computed using estimated (observed) cumulative frequencies $\hat{F}$. These estimates come with a certain amount of error of estimation. This error of estimation is related to the sample size; because in this experiment, the number of trials in the conditions used to compute the bounds is the same, the error of estimation is the same for all conditions. Let's denote the absolute error of estimation for $\hat{F}$ by $\Delta F$ (the exact same argument follows if we use the standard error of estimation $S_{\hat{F}}$ instead of the absolute error of estimation $\Delta F$; Tremblay and Chassé, 1970).

In the Diederich bound, one entry in the min rule is composed of three estimated cumulative probabilities so that using the derivative rule, we have

$$
\Delta \vec{F}=\left|\frac{\partial 3 F}{\partial F}\right| \Delta F=3 A F
$$

The absolute error for one entry in the Diederich bound is three times larger than the absolute error of one cumulative distribution. Some of these errors will be positive so that the true position of the Diederich bound will be overestimated. Likewise, 
some of these errors will be negative, underestimating the true position of the Diederich bound. Overall, the Diederich bound uses a min rule so that the most underestimated position will be preserved. Between the estimated bound and the true bound positions, there may be a small band where the observed empirical data in the triple redundant condition could lie, falsely leading to the conclusion that there is coactivation.

In the Engmann bound, an entry in the max rule is of the form $1-\left(1-F_{i j}(t)\right)\left(1-F_{k}(t)\right)$ so that we have two sources of error estimation. Using the derivative rule, we get

$$
\Delta \bar{F}_{*}=\left|\frac{\partial 1-(1-\bar{F})^{2}}{\partial \bar{F}}\right|_{\Delta F=2(1-\hat{F}) \Delta \hat{F}}
$$

Because $\hat{F}$ is necessarily a probability between 0 and 1 , we have that $0 \leq(1-\hat{F}) \leq 1$ so that in the worst-case scenario, $(1-\hat{F})$ can be replaced by 1 . Hence, we equate

\section{$\mathbf{A} \hat{F}_{*}=\mathbf{2 A} \dot{\vec{F}}$}

The magnitude of the absolute error of estimation for one item in the Engmann bound is a little bit smaller than the corresponding absolute error of estimation in the Diederich bound. However, and this is the most important thing to note, the Engmann bound uses a max rule, so that the estimated bound will most likely overestimate the position of the true bound. With the Engmann bound, the only way that the results in the triple redundant condition can be declared above the bound is that it is above the bound AND above the maximum of two sources of errors. 